\title{
Effect of an Inpatient Geriatric Consultation Team on Functional Outcome, Mortality, Institutionalization, and Readmission Rate in Older Adults with Hip Fracture: A Controlled Trial
}

\author{
Mieke Deschodt, RN, MSN, ${ }^{* \dagger}$ Tom Braes, RN, MSN, ${ }^{* \dagger}$ Paul Broos, MD, PhD, ${ }^{\ddagger}$ An Sermon, MD, ${ }^{\ddagger}$ \\ Steven Boonen, $\mathrm{MD}, \mathrm{PhD}, \stackrel{\mathcal{S}, \ddagger}{\ddagger}$ Johan Flamaing, $\mathrm{MD}, \mathrm{PhD}{ }^{\dagger}$ and Koen Milisen, $\mathrm{RN}, \mathrm{PhD}{ }^{* \dagger}$
}

OBJECTIVES: To evaluate the effect of an inpatient geriatric consultation team (IGCT) on end points of interest in people with hip fracture: length of stay, functional status, mortality, new nursing home admission, and hospital readmission.

DESIGN: Controlled trial based on assignment by convenience.

SETTING: Trauma ward in a university hospital.

PARTICIPANTS: One hundred seventy-one people with hip fracture aged 65 and older.

INTERVENTION: Participants were assigned to a multidisciplinary geriatric intervention $(\mathrm{n}=94)$ or usual care $(\mathrm{n}=77)$ during hospitalization after hip fracture.

MEASUREMENTS: End points were functional status, length of stay, mortality, new nursing home admission, and hospital readmission 6 weeks, 4 months, and 12 months after surgery.

RESULTS: Mean length of stay was $11.1 \pm 5.1$ days in the intervention group and $12.4 \pm 8.5$ days in the control groups $(P=.24)$. Complete adherence to IGCT recommendations was $56.8 \%$. A significant benefit of intervention on functional status in univariate analyses $(P=.02) 8$ days after surgery disappeared in a linear mixed model. Participants with dementia had better functional status in a linear mixed model than those without $(P=.03)$, but this effect was no longer significant after Bonferroni correction for multiple testing. After 6 weeks, 4 months, and 12 months, no between-group differences could be documented for mortality, new nursing home admission, or readmission rate.

CONCLUSION: This trial could not document functional benefits of an IGCT intervention in people with hip frac-

From the ${ }^{*}$ Center for Health Services and Nursing Research, ${ }^{\S}$ Center for Metabolic Bone Diseases, Katholieke Universiteit Leuven, Leuven, Belgium; and Division of Geriatric Medicine and Department of Traumatology,

University Hospitals Leuven, Leuven, Belgium.

Address correspondence to Koen Milisen, Center for Health Services and Nursing Research, Katholieke Universiteit Leuven, Kapucijnenvoer 35/4, B-3000 Leuven, Belgium. E-mail: koen.milisen@med.kuleuven.be

DOI: 10.1111/j.1532-5415.2011.03488.x ture. More research is needed to investigate whether a more-intensive approach with more-direct control over patient management, more-specific recommendations, and more-intense education would be effective. J Am Geriatr Soc 59:1299-1308, 2011.

Key words: geriatric consultation; inpatient; elderly; hip fracture

$T$ he number of older people in Western countries has been increasing over the last decades. ${ }^{1}$ In the near future, the number and proportion of older persons in the population will grow rapidly, with baby boomers now in their 50s and early 60s. ${ }^{2}$ Because individuals aged 65 and older experience three to four times as many hospital days as the general population, healthcare workers will increasingly be involved in caring for older persons, ${ }^{3}$ but specialized healthcare professionals on nongeriatric wards often lack the specific experience and interest required to address the complex needs of older adults effectively. ${ }^{4}$ This "culture and knowledge gap" may have a far-reaching effect on a number of aspects of care. Hospitalized older adults - with their typical frailty profile-are more prone to develop functional decline and more susceptible to cognitive and depressive disorders. ${ }^{5,6}$ Moreover, healthcare professionals often misperceive changes in cognitive and functional status as a normal consequence of aging. This agism stereotype can result (in)directly in negative outcomes, such as an accelerated deterioration of overall health. ${ }^{5,6}$

A systematic approach based on a comprehensive geriatric assessment (CGA) is the best way to detect geriatric problems and to address the complex and specific needs of frail and hospitalized older adults., ${ }^{7,8}$ CGA is defined as "a multidimensional interdisciplinary diagnostic process focused on determining a frail elderly person's medical, psychosocial and functional capabilities in order to develop a 
coordinated and integrated plan for treatment and long-term follow-up."7

One of the geriatric assessment programs currently active in daily clinical practice is the inpatient geriatric consultation team (IGCT). The concept of IGCT reached its "peak attention" in the international literature between 1980 and 2000, but studies reporting on the effects of IGCT have varied widely in terms of design, team composition, and assessment of different outcomes. In a 1990 review, intervention of an IGCT showed positive effects in only three of 13 studies, with possible benefits on mental status, short-term survival, and length of stay, ${ }^{9}$ but more-recent randomized controlled trials (RCTs) that have evaluated the effectiveness of consultation teams have had inconclusive findings. Some of these studies have reported significant improvements in 6-month survival, activities of daily living (ADLs), and rate of hospital readmissions. ${ }^{10-12}$ Others have failed to show significant differences in outcomes between control and intervention groups in terms of mortality, hospital readmission, nursing home admission, length of stay, and hospital charges. ${ }^{13-15}$

Although multidisciplinary IGCTs are considered highly relevant from a practical and clinical perspective, sound and reliable evidence of their effectiveness is lacking. ${ }^{16}$ Moreover, most prior studies of inpatient geriatric consultation did not focus specifically on the acute care of older adults with hip fracture. In this context, the aim of the current study was to test the hypothesis that an IGCT-based intervention would improve ADL functioning and reduce length of stay, mortality, nursing home admission, and hospital readmission in a specific subset of older individuals (individuals consecutively admitted to hospital with a traumatic hip fracture).

\section{METHODS}

\section{Design and Participants}

A single-center, parallel-group trial was conducted in the Leuven University Hospitals (the major university hospital in Belgium; 1,955 beds) from February to December 2007 with follow-ups at 6 weeks, 4 months, and 12 months after inclusion in the study. Verbally competent individuals aged 65 and older consecutively admitted to the emergency department with a traumatic hip fracture were eligible for inclusion. Exclusion criteria were the presence of multiple trauma or metastatic cancer or other known comorbidity expected to reduce the individuals' life expectancy to less than 6 months.

\section{Intervention}

The control group received usual care from the trauma team. Clinical activities in the usual care protocol included evaluation of living conditions before admission by social workers, early mobilization and rehabilitation by the physiotherapist, treatment with anticoagulants and nonopioid pain medication, and a postoperative X-ray of the hip. Participants in the intervention group received usual care as well, but with additional assessment and advice by the IGCT. The IGCT consisted of a geriatrician (JF), three nurses (TB), a social worker, two occupational therapists, and a physiotherapist, all with extensive experience in geriatric care. Other disciplines were available on call. The intervention by the team included four successive steps: CGA, in-depth multidisciplinary evaluation, formal clinical advice and recommendations, and in-hospital follow-up upon request (Appendix 1). Detailed information on the assessment method and instruments and the implementation of the IGCT have been previously reported. ${ }^{17}$

Within 72 hours after the initial comprehensive geriatric assessment or in-depth multidisciplinary evaluation, the IGCT made specific and individualized recommendations in addition to and different from the usual care delivered by the trauma care team. Recommendations were communicated in two ways: a detailed report was included in the participant's electronic file, and recommended courses of action were discussed between the IGCT nurse and the healthcare team of the trauma ward as part of their weekly team meeting. When additional recommendations were made during subsequent hospitalizations, these were added to the initial structured report and discussed with the trauma team.

\section{Variables and Instruments \\ Demographic and Baseline Data}

The following baseline data were collected: age, sex, living situation, type of hip fracture, number of medications, comorbidity, mental status, and confirmed diagnosis of dementia. Comorbidity was assessed according to the Charlson Comorbidity Index. ${ }^{18}$ Mental status was measured using the 12-item Mini-Mental State Examination (MMSE), ${ }^{19}$ the Confusion Assessment Method (CAM), ${ }^{20}$ the Delirium Index (DI), ${ }^{21}$ the Informant Questionnaire on Cognitive Decline in the Elderly (IQ-CODE), ${ }^{22}$ and the 10item Geriatric Depression Scale (GDS). ${ }^{23}$ The diagnosis of dementia was based on the item "dementia" in the Charlson Comorbidity Index. Baseline data were collected before surgery, through participant interview, by screening the medical and nursing files, or both.

\section{Adberence}

The research team systematically and prospectively listed all recommendations made for each participant in the intervention group using a structured check list. As a control measure, these structured checklists were checked retrospectively for correctness and completeness for each participant. To this end, each recommendation was searched for in the participant's electronic file. Adherence was qualified as an order, report, result, or note in the electronic file that indicated that adherence to the recommendation was planned or that the recommendation had been followed. ${ }^{24}$ Adherence was evaluated using three measures: complete, partial, and nonadherence rates. Complete adherence rate was defined as the number of recommendations the team of the nursing ward completely adhered to divided by the number of recommendations that the IGCT made. ${ }^{25}$ To be considered adherent, recommendations had to be initiated within 5 days after communication, unless specified otherwise. Partial adherence rate implied that the participant in the intervention group received only some recommendations or did not receive recommendations in a timely manner, with partial adherence rate defined as the number of recommendations the team partially adhere to 
divided by the number of recommendations made. ${ }^{24}$ Likewise, nonadherence rate was the number of recommendations that the team did not comply with divided by the number of recommendations that the IGCT made. The effect of each specific recommendation was further estimated by multiplying its frequency with the complete adherence rate. ${ }^{26}$ For instance, the recommendation to perform additional blood tests was made in $40.1 \%$ of the intervention group, with $63.6 \%$ complete adherence. Thus, in $63.6 \%$ of $40.1 \%$, or $25.5 \%$ of the intervention group, additional blood tests were performed based on the recommendations of the IGCT.

\section{Outcome Measures}

The primary end point was in-hospital functional status. Functional status was measured using the 6-item Katz Index of activities of daily living (ADLs). ${ }^{27}$ Dependence in bathing, dressing, transfer, toileting, incontinence, and feeding was assessed on a 3 -point scale $(1=$ independent, $2=$ partially dependent, 3 = fully dependent), resulting in a continuous total score ranging from 6 to 18 .

Additional outcomes were length of stay and mortality, unplanned readmission, and new nursing home admission rate. Length of stay during the acute phase was defined as the number of postoperative days on the trauma ward until discharge or death. The day after surgery was defined as the first postoperative day. For participants transferred to a geriatric or rehabilitation ward, the total length of stay was defined as the number of days on the trauma ward and the number of days on the geriatric or rehabilitation ward until discharge or death.

Mortality was defined as the number of events (death) from the first day after surgery until 6 weeks, 4 months, and 12 months.

An unplanned readmission was defined as a subsequent or repeat hospitalization that followed the initial hospitalization and could not be foreseen at the time of discharge, regardless of whether readmission occurred on a nonemergency or emergency basis. ${ }^{28}$ It was measured 6 weeks, 4 months, and 12 months postoperatively.

A new nursing home admission was defined as the number of events (new admissions in a nursing home) from the initial hospitalization until 6 weeks, 4 months, and 12 months postoperatively. By definition, new nursing home admissions were measured only in participants who had not been living in a nursing home before the hip fracture occurred.

\section{Procedure}

Participants were recruited consecutively in the emergency department. Inclusion and exclusion criteria were checked, and informed consent was obtained. Functional status and GDS scores of eligible participants were assessed retrospectively for up to 2 weeks before admission. For cognitively impaired participants, premorbid status was assessed by interviewing a proxy or relative. Baseline characteristics (e.g., age, sex, living situation, number of medications, and type of fracture), cognitive status (MMSE, CAM, DI, and IQ-CODE), and Charlson Comorbidity Index were assessed within the first 24 hours after admission and before surgery. On the first, third, fifth, and eighth day after surgery, functional status and mortality were evaluated.
From 8 days after surgery on - and depending on the participant's length of stay-functional status and mortality were assessed weekly (e.g., day 15, day 22). Discharge destination, length of stay, and mortality were assessed when the participant was scheduled for discharge. Six weeks, 4 months, and 12 months after the index admission, the participant (or relatives in case of cognitive impairment) were interviewed over the telephone, and a selection of questions and self-completion instruments (ADLs, instrumental ADLs) was mailed to assess functional status, mortality, living situation, and unplanned readmissions.

A doctoral student (TB) and his supervisor (KM) trained all three data nurses who gathered information and assessed the participants. The Medical Ethics Committee of the Leuven University Hospitals approved the study. After written and oral information was provided, informed (proxy) consent was obtained for every participant before inclusion. Consent continued to be checked at the different assessment times.

\section{Sample Size}

According to a power analysis using a two-tailed test of significance with an alpha of 0.05 and a beta of 0.10 to detect a difference of two points in ADL functional status (standard deviation $=4$ ), a sample size of 70 participants was required in each group. ${ }^{29}$ Taking into account a possible dropout rate of approximately $15 \%$ to $20 \%$ after 1 year of follow-up, this corresponded to 80 to 85 participants in each group.

\section{Assignment}

All eligible participants were allocated to one of the two trauma wards of the hospital (one intervention and one control ward). The assignment of participants was imbedded in the normal procedures of the hospital's admission service. After admission to the emergency department, the admission service determined on which of the two trauma wards the participant would be hospitalized, a decision based solely on the availability of beds.

\section{Blinding}

Neither participants, healthcare professionals on the trauma wards, nor members of the IGCT could be blinded to the intervention, because it was based on face-to-face contact between participants and the IGCT. Collected data were analyzed blinded to the group allocation of the participant.

\section{Statistical Methods}

Data gathered as part of the study were coded and analyzed anonymously. Descriptive analyses were done for the control and intervention groups; continuous variables were expressed as means and interquartile ranges and categorical variables as number of cases and percentages. The effect of the intervention on ADL functioning was tested in a linear mixed model for repeated measures. All ADL measurements were included to fit a linear model, with baseline ADL score, group, time, and an interaction between group and time as explanatory variables. A transformation of time (1/log (time)) led to the best model fit. In addition to analyses of the available ADL scores of all participants, 
different mortality analyses were done: one with participants who died was excluded from the analyses and a second one in which participants who died were given the worst possible (maximum) ADL score once they had died. All analyses were repeated for the following subgroups of participants: participants with a baseline ADL score of 6 (independent for all aspects), 7 or greater (dependence for at least one aspect), and 12 or greater (most dependent participants) and participants with and without dementia.

All analyses were performed on an intention-to-treat principle. Univariate analyses were performed using SPSS version 17.0. (SPSS, Inc., Chicago, IL); the linear mixed model was performed using the MIXED procedure in SAS version 9.2 (SAS Institute, Inc., Cary, NC). Statistical significance was established as $P<.05$, with all tests being two-tailed.

\section{RESULTS}

\section{Study Population}

During the study, 287 people with hip fracture were consecutively admitted to the study hospital; 116 were excluded from the study (Figure 1). All 171 consenting participants were successfully assigned to the intervention $(n=94)$ or control group $(n=77)$. Table 1 compares prefracture participant characteristics of both groups. There were no statistically significant differences in any of the baseline characteristics examined.

\section{Content of Recommendations Made by the Geriatric Consultation Team}

Recommendations were made for 79 of the 94 participants in the intervention group $(84.0 \%)$. No recommendations were given to 15 participants because no need for additional advice on top of usual care could be identified. Of the 338 recommendations made for these 79 participants, adherence could not be determined for nine recommendations, leaving 329 recommendations for study (97.3\%). The occupational therapist had made the nine recommendations with unknown adherence, suggesting walking aids or adapted footgear. The mean number of recommendations per participant was $4.3 \pm 2.1$, ranging from one to 10 . Complete and partial adherence rates were 56.8\% (187/ $329)$ and $10.6 \%$ (35/329), respectively. The healthcare team on the trauma ward did not comply with 107 (32.5\%) recommendations.

Table 2 gives a structured overview of the recommendations that the IGCT made, reporting their frequency, adherence rates, and effect. The frequency of particular

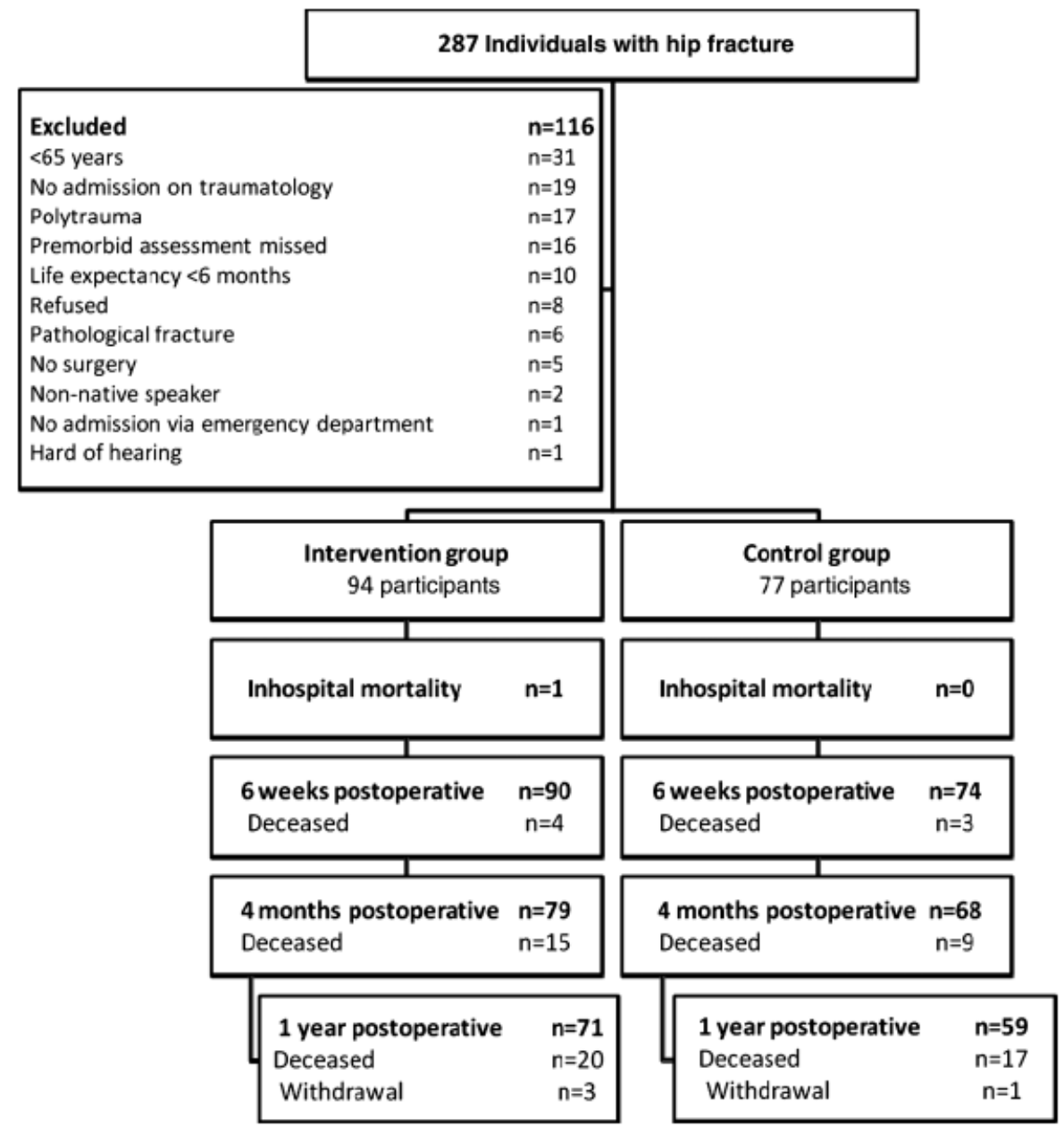

Figure 1. Flowchart of individuals with hip fracture. 


\begin{tabular}{|c|c|c|c|}
\hline Characteristic & Intervention $(n=94)$ & Control $(n=77)$ & $P$-Value \\
\hline Age, mean \pm SD & $80.4 \pm 7.0$ & $81.1 \pm 7.2$ & $.49^{*}$ \\
\hline Female, n (\%) & $69(73.4)$ & $57(74.0)$ & $.93^{\dagger}$ \\
\hline Living situation, n (\%) & & & $.78^{\dagger}$ \\
\hline At home alone & $33(35.1)$ & $22(28.6)$ & \\
\hline At home with others & $32(34.0)$ & $31(40.3)$ & \\
\hline Nursing home & $26(27.7)$ & $20(26.0)$ & \\
\hline Other (service flat, cloister) & $3(3.2)$ & $4(5.2)$ & \\
\hline Type of surgery, n (\%) & & & $.38^{\dagger}$ \\
\hline Proximal femur nail antirotation & $46(48.9)$ & $43(55.8)$ & \\
\hline Bi-articular hip prosthesis & $28(29.8)$ & $24(31.2)$ & \\
\hline Total hip prosthesis & $10(10.6)$ & $3(3.9)$ & \\
\hline Other & $10(10.6)$ & $7(9.1)$ & \\
\hline Number of medications taken at home, mean \pm SD & $5.8 \pm 3.9$ & $5.8 \pm 3.2$ & $.98^{*}$ \\
\hline Katz activity of daily living score, mean \pm SD (range 6-18) & $8.7 \pm 3.3$ & $9.3 \pm 3.2$ & $.07^{\ddagger}$ \\
\hline Dementia, $n(\%)$ & $16(17.2)$ & $18(24.0)$ & $.28^{\dagger}$ \\
\hline $\begin{array}{l}\text { Informant Questionnaire on Cognitive Decline in the Elderly score, } \\
\text { mean } \pm \text { SD (range 1-5) }\end{array}$ & $3.6 \pm 0.7$ & $3.6 \pm 0.8$ & $.62^{\ddagger}$ \\
\hline Charlson Comorbidity Index, mean \pm SD & $2.4 \pm 2.1$ & $2.1 \pm 1.9$ & $.28^{\ddagger}$ \\
\hline Mini-Mental State Examination score, mean \pm SD (range 0-12) & $7.3 \pm 3.9$ & $7.2 \pm 3.9$ & $.96^{\ddagger}$ \\
\hline Confusion Assessment Method, $\mathrm{n}(\%)$ & & & $.46^{\dagger}$ \\
\hline No delirium & $73(78.4)$ & $56(73.7)$ & \\
\hline Delirium & $20(21.6)$ & $20(26.3)$ & \\
\hline Missing & 1 & 1 & \\
\hline Delirium Index, mean \pm SD (range 0-21) & $4.8 \pm 4.0$ & $5.2 \pm 4.4$ & $.62^{\ddagger}$ \\
\hline Geriatric Depression Scale score, mean \pm SD (range $0-10$ ) & $2.8 \pm 2.4$ & $2.5 \pm 2.4$ & $.30^{\ddagger}$ \\
\hline
\end{tabular}

*Unpaired $t$-test.

${ }^{\dagger}$ Chi-square test.

${ }^{\ddagger}$ Mann-Whitney $U$-test.

$\mathrm{SD}=$ standard deviation

recommendations and their adherence rates varied widely. Recommendations frequently made and overall well adhered to $(>80 \%)$ were screening for and treatment of low hematocrit or hemoglobin and organizing a transfer to a rehabilitation center. The recommendations least adhered to $(<45 \%)$ were planning an appointment for bone densitometry, performing additional cognitive testing in the geriatric day hospital, planning an appointment for a fall risk assessment and fall preventive measures in the geriatric day hospital, eliminating redundant medication, and prescribing calcium and vitamin D supplements upon discharge. Recommendations with the highest effect (balancing frequency and complete adherence) were screening for and treatment of low hematocrit or hemoglobin $(27.2 \%)$, organizing a transfer to a rehabilitation center $(26.0 \%)$, and performing additional blood tests $(25.5 \%)$.

\section{Care Received According to Intervention and Control Group}

Table 3 compares the care given to the intervention and control groups. No statistically significant differences were found, except that the intervention group received more interventions from the occupational therapist $(P<.001)$ and more opioid pain medication $(P=.004)$.

\section{Effect of the Intervention on Outcome Parameters Functional Status}

In the univariate analysis, participants in the intervention group were significantly less dependent 8 days after surgery than controls (ADL score $12.0 \pm 2.7$ vs $12.9 \pm 2.5$, $P=.02$ ), but ADL status was not significantly different at any of the other time points (Table 4). The linear mixed model for repeated measures, with two ways of treating missing due to death, showed no difference between the groups on ADL scores.

Subanalyses were performed in completely independent participants based on ADL status at baseline (ADL score before admission of 6) and dependent participants (ADL score $\geq 7$ and $\geq 12$ ), but no effect of the intervention $(P=.31$ to .99$)$ could be documented in any of the subgroups of participants. (Data not shown, but available upon request.) Similarly, subanalyses were performed in participants with and without dementia. In participants without dementia (mean IQ-CODE $3.4 \pm 0.05$ ), there was no evidence of a difference in ADL functioning between the intervention and control groups. In the 34 participants with dementia at baseline (mean IQ-CODE 4.6 \pm 0.11 , $P<.001$ ), no significant difference in baseline ADL functioning was observed between the groups $(P=.25)$, although a significant effect in favor of the intervention 
Table 2. Structured Overview of the Recommendations Given by the Inpatient Geriatric Consultation Team $(\mathrm{N}=329)$

n (\%)

Recommendation

Recommended* $^{*} \quad$ Complete Adherence $^{\dagger} \quad$ Partial Adherence $^{\ddagger} \quad$ Nonadherence Effect, \%§

Fluid and electrolyte balance

Treat fluid overload or dehydration detected

$1(1.2)$

$1(100)$

1.2

by examination or blood test

Treat electrolyte imbalance

$8(9.9)$

$7(87.5)$

$1(12.5)$

8.7

Additional tests and investigations

Perform additional blood tests

$33(40.1)$

21 (63.6)

$5(15.2)$

7 (21.2)

25.5

2 (33.3)

$1(16.7)$

Perform additional technological

$6(7.4)$

computed tomography scan, echography)

Prevention, early detection, and treatment of postoperative complications

Myocardial infarction or

ischemia - electrocardiogram — cardiac

enzymes

Supraventricular arrhythmias or atrial

fibrillation - appropriate rate control,

electrolyte adjustments, anticoagulation

Pneumonia, chronic obstructive pulmonary

disease - screening treatment including

chest therapy

Pulmonary embolus - appropriate

anticoagulation

Screening for and treatment of urinary tract infection

2 (2.5)

$2(100)$

$3(50.0)$

$2(66.7)$

$1(33.3)$

\section{Adequate oxygen delivery}

Screening for and treatment of low

$26(32.1)$

22 (84.6)

4 (15.4)

27.2

hematocrit or hemoglobin (e.g., transfusion)

Supplemental oxygen to keep saturation

$6(7.4)$

4 (66.7)

$1(16.7)$

$1(16.7)$

4.9

, preferably $>95 \%$

\section{Medication}

Discontinue or minimize benzodiazepines,

anticholinergics, antihistamines

Eliminate drug interactions, adverse effects,

modify drugs accordingly

Eliminate medication redundancies

15 (18.5)

$6(40.0)$

2 (13.3)

7 (46.7)

7.4

Start up new medication

$10(12.3)$

$7(70.0)$

$1(10.0)$

$2(20.0)$

8.6

$21(25.9)$

7 (33.3)

$1(4.8)$

$13(61.9)$

$2(11.1)$

$4(22.2)$

8.6

$17(21.0)$

$11(64.7)$

$1(33.3)$

$2(66.7)$

13.6

Regulation of bowel or bladder function

Bowel function: bowel movement by

3 (3.7)

$2(66.7)$

$1(33.3)$

1.2

Remove urinary catheter by postoperative

$1(100)$

$1(1.2)$

(100)

established incontinence

Nutritional intake

Call in dietitian (based on results mini

$1(1.2)$

$1(100)$

0

nutritional assessment)

Osteoporosis and falls

Prescribing calcium and vitamin D

supplements

Recurrent falls: appointment at falls clinic

Planning appointment for bone densitometry

$26(32.1)$

$8(30.8)$

$3(11.5)$

$15(57.7)$

9.9

Cognitive status

Pharmacological treatment of agitated delirium (according to hospital guideline)

Additional cognitive testing in geriatric day

$8(9.9)$

$1(12.5)$

$1(12.5)$

$6(75.0)$

1.2

$11(13.3)$

$11(100)$

0

clinic

Information or sensibilization when dementia

suspected

$14(17.3)$

$2(14.3)$

$8(57.1)$

4 (28.6)

2.5

7 (8.6)

$7(100)$

0

3 (3.7)

$2(66.7)$

1 (33.3)

2.5

Call in gerontopsychiatrist

$11(13.6)$

$8(72.7)$

3 (27.3)

9.9 
Table 2. (Contd.)

\begin{tabular}{|c|c|c|c|c|c|}
\hline \multirow[b]{2}{*}{ Recommendation } & \multicolumn{4}{|c|}{ n (\%) } & \multirow[b]{2}{*}{ Effect, \%§ } \\
\hline & Recommended* & Complete Adherence $^{\dagger}$ & Partial Adherence ${ }^{\ddagger}$ & Nonadherence & \\
\hline \multicolumn{6}{|l|}{ Living situation and discharge destination } \\
\hline $\begin{array}{l}\text { Organizing temporary/definitive alternative to } \\
\text { living at home }\end{array}$ & $7(8.6)$ & $7(100)$ & & & 8.6 \\
\hline Organizing transfer to rehabilitation center & $25(30.9)$ & $21(84.0)$ & $2(8.0)$ & $2(8.0)$ & 26.0 \\
\hline Evaluating burden informal caregiver & $5(6.2)$ & $5(100)$ & & & 6.2 \\
\hline \multicolumn{6}{|l|}{ Preventive measures and nursing recommendations } \\
\hline Prevention of pressure ulcers & $2(2.5)$ & $1(50.0)$ & $1(50.0)$ & & 1.3 \\
\hline Nursing recommendations & $3(3.7)$ & $2(66.7)$ & $1(33.3)$ & & 2.5 \\
\hline \multicolumn{6}{|l|}{ Rehabilitation } \\
\hline $\begin{array}{l}\text { Call in other disciplines (neurology, speech } \\
\text { therapist, dermatology, ophthalmology, } \\
\text { endocrinology) }\end{array}$ & $8(9.9)$ & $4(50.0)$ & $1(12.5)$ & $3(37.5)$ & 5.0 \\
\hline Transfer to emergency unit & $1(1.2)$ & $1(100)$ & & & 1.2 \\
\hline $\begin{array}{l}\text { Clear documentation of do-not-resuscitate } \\
\text { order }\end{array}$ & $1(1.2)$ & & & $1(100)$ & 0 \\
\hline
\end{tabular}

* Intervention patients in whom the recommendation was made.

${ }^{\dagger}$ Recommendations with which the care team of the intervention group completely adhered to divided by the number of recommendations made.

${ }^{\ddagger}$ Recommendations with which the care team of the intervention group partially adhered to divided by the number of recommendations made.

${ }^{\S}$ Patients in the intervention group in which the recommendations made by the geriatric consultation team are performed completely and correctly.

group $(\mathrm{n}=16)$ was found when using a linear model on surviving participants only $(P=.048)$ and on all participants when participants who had died were given a maximum ADL score of $18(P=.03)$. This result was not found in the linear model analyses on all participants $(P=.17)$. Including the premorbid living situation as a covariate in the model did not alter the results. (Data not shown, but available upon request.)

\section{Length of Stay}

There was no statistically significant difference between the intervention and control groups in mean length of stay on the trauma ward $(11.1 \pm 5.1$ days vs $12.4 \pm 8.5$ days, $P=.24$ ) or for the number of participants transferred to a geriatric or rehabilitation unit $(30,31.9 \%$ vs $26,33.8 . \%$; $P=.80$ ). Mean total length of stay for participants transferred to a geriatric or rehabilitation unit was not

\begin{tabular}{|c|c|c|c|}
\hline \multirow[b]{2}{*}{ Clinical Activity } & \multicolumn{2}{|c|}{$\mathrm{n}(\%)$} & \multirow[b]{2}{*}{$P$-Value } \\
\hline & Intervention $(\mathrm{n}=94)$ & Control $(n=77)$ & \\
\hline Preoperative electrocardiogram & $92(97.9)$ & $74(96.1)$ & .66 \\
\hline Postoperative hip X-ray & $92(97.9)$ & $76(98.7)$ & .99 \\
\hline Postoperative anticoagulation & $94(100)$ & $77(100)$ & 1.00 \\
\hline Nonopioid pain medication & $94(100)$ & $76(98.7)$ & .45 \\
\hline Opioid pain medication & $86(91.5)$ & $58(75.3)$ & .004 \\
\hline Calcium and vitamin $\mathrm{D}$ & $21(22.3)$ & $21(27.3)$ & .46 \\
\hline Blood transfusion & $40(42.6)$ & $38(49.4)$ & .38 \\
\hline Early mobilization and rehabilitation & $64(68.1)$ & $56(72.7)$ & .51 \\
\hline Additional blood tests $(>5)$ & $40(42.6)$ & $30(39.0)$ & .64 \\
\hline Additional electrocardiogram $(>1)$ & $21(22.3)$ & $20(26.0)$ & .58 \\
\hline Additional technical investigations & $46(48.9)$ & $41(53.2)$ & .58 \\
\hline Occupational therapy & $65(69.1)$ & $32(41.6)$ & $<.001$ \\
\hline Consult social work & $75(79.8)$ & $65(84.4)$ & .43 \\
\hline Consult geropsychiatrist & $11(11.7)$ & $9(11.7)$ & 1.00 \\
\hline Consult other disciplines & $25(26.6)$ & $23(29.9)$ & .64 \\
\hline
\end{tabular}


significantly different $(P=.90)$ between the intervention (56.3 \pm 43.7 days) and control (55.1 \pm 25.5 days) groups either.

\section{Mortality, Readmission Rate, and New Nursing Home Admission}

No statistically significant difference in all-cause mortality rate was found 6 weeks, 4 months, or 12 months after surgery. The number of participants with unplanned readmission to the hospital did not differ significantly between the two groups at 6 weeks $(P=.47), 4$ months $(P=.47)$, or 12 months $(P=.77)$ postoperatively, nor did the mean total number of readmission days (intervention: $20.9 \pm 17.6$ days vs control: $18.8 \pm 13.1$ days; $P=.64$ ). The proportion of participants newly admitted to a nursing home was not significantly different at any time point either (Table 4).

\section{DISCUSSION}

In this study in older adults with hip fracture, no impact of an IGCT was found on length of stay, mortality, hospital readmission, or the need for institutional care. One week after surgery, the intervention group had significant improvement in ADL functioning in univariate analyses, but the effect disappeared in linear mixed model analyses. The only benefit that persisted in the linear mixed model analyses was improved functional status in participants with dementia, although this apparent benefit should be interpreted with caution. To address the complexity of missing data, three different models (linear model on all participants and two ways of treating missing values due to death) were fitted, and the benefit in participants with dementia was not consistently observed across all models. Similarly, when performing a Bonferroni correction to adjust for multiple testing, the effect was no longer significant. This study should be interpreted as negative, despite the focus on a well-defined frail population with advanced age and functional impairment.

Methodological problems may have contributed to the lack of a difference between the control and intervention groups. First, it cannot be excluded that the care given on both wards in the study setting - a tertiary university hospital - was already relatively effective. With more than 300 people with hip fracture admitted to the trauma wards each year, healthcare professionals have experience with frail older adults with hip fracture. The fact that physical therapy was given as standard care to all participants may also have reduced any effect of the intervention on ADL functioning. Second, lack of adherence to the IGCT recommendations is likely to have contributed to the negative findings. With approximately one-third of the recommendations not adhered to and approximately $10 \%$ only partially adhered to, in line with previous studies, ${ }^{24-26}$ there is room for improvement. Of particular concern is that recommendations to refer for comprehensive fall assessment and prevention and to supplement participants with calcium and vitamin $\mathrm{D}$ were hardly adhered to at all, despite their proven efficacy in older adults. ${ }^{30,31}$ In addition, when comparing the actual care given of the intervention and control groups, a significant difference was found in the percentage of participants receiving occupational therapy. Although ADL functioning is one of the main focuses of occupational ther- apists, no significant differences were observed in ADLs between the groups. This could reflect the lack of intensity of the intervention because the occupational therapist of the IGCT intervened only one or two times per participant during the hospitalization period but could also be due to the lack of continuity because the adherence rate to the advice given or the therapy started was low. Finally, participants, IGCT members, and healthcare professionals on the trauma ward could not be blinded to the intervention, although observer bias was minimized by using structured questionnaires and scales to evaluate the outcomes of interest.

Despite limitations intrinsic to the design of the study, the findings do not support this model of a geriatric consultation team as an effective tool to improve outcomes. Part of the appeal of the concept of IGCT is that IGCTs have some important advantages over increasing the capacity of Acute Care for the Elderly units; they are less expensive and more flexible, can be implemented within a short period of time, and can reach a larger number of individuals, ${ }^{13,32}$ but a number of studies, including the present one, have failed to demonstrate the effectiveness of IGCTs, at least on functional status, length of stay, readmission rate, or need for institutionalization. ${ }^{11-13}$ Although these parameters are important in this population, it is possible that the intervention would significantly affect more-clinical outcomes, such as delirium and pain. After all, significantly more intervention participants than controls received occupational therapy (cognitive interventions included) and pain medication. Future research should therefore investigate whether the IGCT model as described in this article affects such outcomes. More importantly, future studies should investigate whether and to what extent alternative IGCT approaches are more effective. Instead of an advisory model based on recommendations, the multidisciplinary IGCT should probably be used more directly in management of older adults on nongeriatric wards. Bedside education by the IGCT, to familiarize healthcare workers on nongeriatric wards with the specifics of geriatric care and the expression and implications of the major geriatric syndromes (e.g., falls, delirium, depression, dementia, sleeping disorders), could make a difference. An educational unit-centered model, instead of a recommendation-based patient-centered model, would also ultimately allow the IGCT to reach out to more individuals by transferring geriatric expertise to nongeriatric wards. In addition, more attention should be given to communication and collaboration between healthcare workers across settings (e.g., hospital staff, but also caregivers at home and in long-term care facilities). To optimize outcomes in older adults, any IGCT model should affect not only in-hospital care, but follow-up care as well (e.g., referral for specific assessments after discharge, organizing specific support after hospitalization). ${ }^{33}$ This may be particularly important to reduce the risk of readmission to hospital or the need for institutional care.

Based on these negative findings, in older persons with a recent hip fracture, a recommendation-based and patientcentered IGCT model has no significant effect on major outcome parameters such as functional status and length of stay. Future studies should address the effectiveness of an educational approach that allows the IGCT to have more direct control over patient management on nongeriatric wards. 


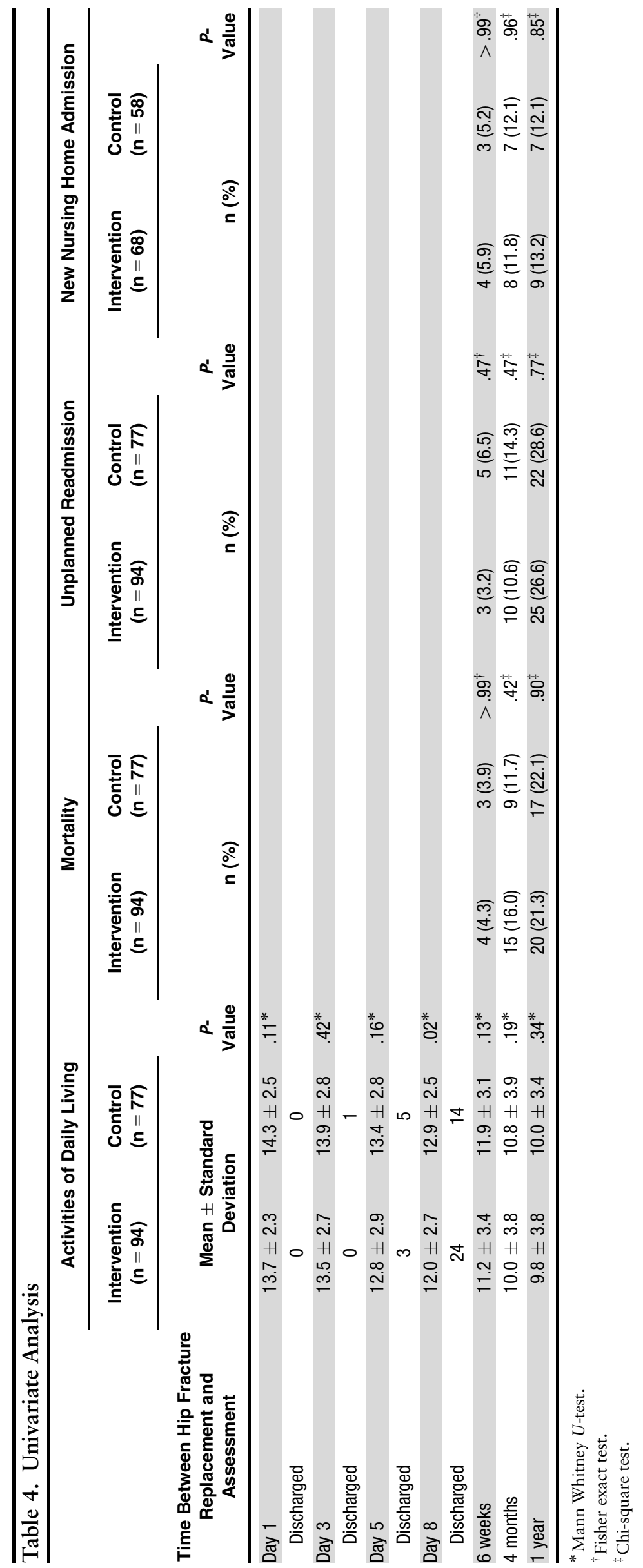




\section{ACKNOWLEDGMENTS}

The authors gratefully acknowledge the input of the geriatric consultation team; the nursing staff of the two participating trauma wards, Walter Pelemans, Lon Holtzer, An Veris, Jan Ampe, and Karine Philippaerts; and all consenting participants. We also thank research assistants Luk Bruyneel, Luc Bosschaerts, Sigrid Aerts, and Katrien Dierickx and the Leuven Biostatistics and Statistical Bioinformatics Center.

Conflict of Interest: The authors have no conflict of interest. Steven Boonen is senior clinical investigator of the Fund for Scientific Research-Flanders, Belgium and holder of the Leuven University Chair in Gerontology and Geriatrics.

Author Contributions: Study concept and design: T. Braes, J. Flamaing, K. Milisen. Statistical analysis: M. Deschodt. Interpretation of the data: M. Deschodt, T. Braes, A. Sermon, S. Boonen, J. Flamaing, K. Milisen. Drafting of the manuscript: M. Deschodt, T. Braes, K. Milisen. Critical revision of the manuscript: M. Deschodt, T. Braes, P. Broos, A. Sermon, S. Boonen, J. Flamaing, K. Milisen. Supervision: J. Flamaing, K. Milisen.

Sponsor's Role: None.

\section{REFERENCES}

1. National Center for Health Statistics. Healthcare in America, trends in utilization, No. 2004-1031 Ed. 2004.

2. DeFrances CJ, Lucas CA, Buie VC et al. National hospital discharge survey. Nat Health Stat Rep 2008;30:1-20.

3. AHA Trendwatch (by The Lewin Group), American Hospital Association Volume 3, Number 3, 2001.

4. Milisen K, Schuurmans M, Hayes N. Urgent need for leadership in nursing care for older persons. Appl Nurs Res 2006;19:171-172.

5. Pedone C, Ercolani S, Catani M et al. Elderly patients with cognitive impairment have a high risk for functional decline during hospitalization: The GIFA study. J Gerontol A Biol Sci Med Sci 2005;60A:1576-1580.

6. Sands LP, Yaffe K, Covinsky K et al. Cognitive screening predicts magnitude of functional recovery from admission to 3 months after discharge in hospitalized elders. J Gerontol A Biol Sci Med Sci 2003;58A:37-45.

7. Rubenstein LZ, Stuck AE, Siu AL et al. Impacts of geriatric evaluation and management programs on defined outcomes: Overview of the evidence. J Am Geriatr Soc 1991;39:8S-16S.

8. Stuck AE, Siu AL, Wieland GD et al. Comprehensive geriatric assessment: A meta-analysis of controlled trials. Lancet 1993;342:1032-1036.

9. Winograd $\mathrm{CH}$, Stearns C. Inpatient geriatric consultation. Challenges and benefits. J Am Geriatr Soc 1990;38:926-932.

10. Hogan DB, Fox R. A prospective controlled trial of a geriatric consultation team in an acute-care hospital. Age Ageing 1990;19:107-113.

11. Thomas DR, Brahan R, Haywood BP. Inpatient community-based geriatric assessment reduces subsequent mortality. J Am Geriatr Soc 1993;41: 101-104.

12. Cohen HJ, Feussner JR, Weinberger $M$ et al. A controlled trial of inpatient and outpatient geriatric evaluation and management. N Engl J Med 2002;346: 905-912.

13. Winograd $\mathrm{CH}$, Gerety $\mathrm{MB}$, Lai NA. A negative trial of inpatient geriatric consultation. Lessons learned and recommendations for future research. Arch Intern Med 1993;153:2017-2023.

14. Fretwell MD, Raymond PM, McGarvey ST et al. The Senior Care Study. A controlled trial of a consultative/unit-based geriatric assessment program in acute care. J Am Geriatr Soc 1990;38:1073-1081.

15. Kircher TTJ, Womstall H, Muller PH et al. A randomized trial of a geriatric evaluation and management consultation services in frail hospitalized patients. Age Ageing 2007;36:36-42.
16. Agostini JV, Baker DI, Inouye SK et al. Multidisciplinary geriatric consultation services. In: Shojania KG, Duncan BW, McDonald KM et al., editors. Evidence Report/Technology Assessment 2001, 43. AHRQ Publication 01E058. Rockville MD: Department of Health \& Human Services, 2001, pp 313-321.

17. Braes T, Flamaing J, Pelemans W et al. Geriatrics on the run: Rationale, implementation and preliminary findings of a Belgian internal liaison team. Acta Clin Belg 2009;64:384-392.

18. Charlson ME, Pompei P, Ales KL et al. A new method of classifying prognostic comorbidity in longitudinal studies: Development and validation. J Chronic Dis $1987 ; 40: 373-383$

19. Braekhus A, Laake K, Engedal K. The Mini-Mental State Examination: Identifying the most efficient variables for detecting cognitive impairment in the elderly. J Am Geriatr Soc 1992;40:1139-1145.

20. Inouye SK, van Dyck CH, Alessi CA et al. Clarifying confusion: The Confusion Assessment Method. A new method for detection of delirium. Ann Intern Med 1990;113:941-948.

21. McCusker J, Cole MG, Dendukuri $\mathrm{N}$ et al. The Delirium Index, a measure of the severity of delirium: New findings on reliability, validity, and responsiveness. J Am Geriatr Soc 2004;52:1744-1749.

22. Jorm AF, Scott R, Cullen JS et al. Performance of the Informant Questionnaire on Cognitive Decline in the Elderly (IQCODE) as a screening test for dementia. Psychol Med 1991;21:785-790.

23. Almeida OP, Almeida SA. Short versions of the Geriatric Depression Scale: A study of their validity for the diagnosis of a major depressive episode according to ICD-10 and DSM-IV. Int J Geriatr Psychiatry 1999;14:858-865.

24. Allen CM, Becker PM, McVey LJ et al. A randomized, controlled clinical trial of a geriatric consultation team. Compliance with recommendations. JAMA 1986;255:2617-2621.

25. Inouye SK, Bogardus ST Jr., Williams CS et al. The role of adherence on the effectiveness of nonpharmacologic interventions: Evidence from the Delirium Prevention Trial. Arch Intern Med 2003;163:958-964.

26. Marcantonio ER, Flacker JM, Wright RJ et al. Reducing delirium after hip fracture: A randomized trial. J Am Geriatr Soc 2001;49:516-522.

27. Katz S, Akpom CA. Index of ADL. Med Care 1976;14:116-118.

28. Landrum L, Weinrich S. Readmission data for outcomes measurement: Identifying and strengthening the empirical base. Q Manage Health Care 2006; 15:83-85.

29. Faul F, Erdfelder E, Lang AG et al. G*Power 3: A flexible statistical power analysis program for the social, behavioral, and biomedical sciences. Beh Res Methods 2007;39:175-191.

30. Bischoff-Ferrari HA, Willett WC, Wong JB et al. Prevention of nonvertebral fractures with oral vitamin $\mathrm{D}$ and dose dependency. A meta-analysis of randomized controlled trials. Arch Intern Med 2009;169:551-561.

31. Jennings LA, Auerbach AD, Maselli J et al. Missed opportunities for osteoporosis treatment in patients hospitalized for hip fracture. J Am Geriatr Soc 2010;58:650-657.

32. Hogan DB. Impact of geriatric consultation services for elderly patients admitted to acute care hospitals. Can J Aging 1990;9:35-44.

33. Gray L. Geriatric consultation: Is there a future? Age Ageing 2007;36:1-2.

\section{SUPPORTING INFORMATION}

Additional Supporting Information may be found in the online version of this article:

Appendix S1. Flowchart Outlining the Interventions Performed by the Inpatient Geriatric Consultation Team (IGCT).

Please note: Wiley-Blackwell is not responsible for the content or functionality of any supporting materials supplied by the authors. Any queries (other than missing material) should be directed to the corresponding author for the article. 